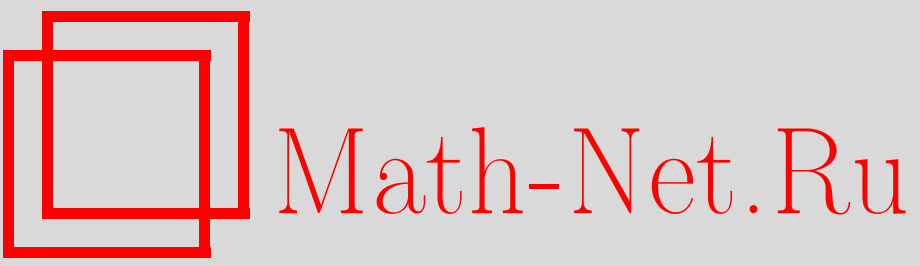

С. М. Ташпулатов, Исследование спектра оператора энергии двухмагнонной системы в одномерном анизотропном ферромагнетике Гейзенберга со взаимодействием до вторых соседей, ТМФ, 1996, том 107, номер 1, 155-161

DOI: https://doi.org/10.4213/tmf1145

Использование Общероссийского математического портала Math-Net.Ru подразумевает, что вы прочитали и согласны с пользовательским соглашением

http: //www.mathnet.ru/rus/agreement

Параметры загрузки:

IP : 54.198 .55 .26

26 апреля 2023 г., $16: 16: 24$ 


\section{ИССЛЕДОВАНИЕ СПЕКТРА ОПЕРАТОРА ЭНЕРГИИ ДВУХМАГНОННОЙ СИСТЕМЫ В ОДНОМЕРНОМ АНИЗОТРОПНОМ ФЕРРОМАГНЕТИКЕ ГЕЙЗЕНБЕРГА СО ВЗАИМОДЕЙСТВИЕМ ДО ВТОРЫХ СОСЕДЕЙ}

Рассматривается физическая система, состоящая из двух магнонов в одномерном анизотропном ферромагнетике Гейзенберга со взаимодействием до вторых соседей. Изучаются спектр и связанные состояния (определение см. ниже) гамильтониана системы. Установлено, что в некоторых случаях у гамильтониана системы возникает или исчезает дополнительное связанное состояние по сравнению с изотропным случаем.

В работах [1-3] изучаются спектральные свойства гамильтониана двухмагнонной системы в ферромагнетике Гейзенберга со взаимодействием ближайших соседей. В этих работах доказано, что в одномерном случае гамильтониан системы обладает не более чем одним связанным состоянием. В работе [3] доказано, что гамильтониан двухмагнонной системы в $d$-мерном пространстве может иметь $0,1,2, \ldots, d$ связанных состояний.

Задача изучения связанных состояний гамильтониана двухмагнонной системы получила дальнейшее развитие в работах [4-7], где рассматриваются системы с ближайшим и вторым взаимодействием.

Изучение спектральных свойств гамильтониана двухмагнонной системы в случае анизотропного ферромагнетика Гейзенберга представляет чрезвычайный интерес для квантовой механики, квантовой физики и физики твердого тела. Трудности, возникаюшие в анизотропном случае по сравнению с изотропным, заключаются в том, что здесь появляются дополнительные параметры, при изменении которых картина энергетического спектра существенно меняется. В настояшей работе изучаются спектр и связанные состояния гамильтониана дву хмагнонной системы в случае $X X Z$-модели [8] со взаимодействием ближайших и вторых соседей, когда полный квазиимпульс системы $\Lambda$ принимает значения $\pi, \frac{\pi}{2}$ и 0 . Для этих значений $\Lambda$ установлено, что при некоторых соотношениях между параметрами гамильтониана возникает одно дополнительное связанное состояние системы или оно исчезает по сравнению с изотропным случаем. Для остальных же значений полного квазиимпульса $\Lambda$ установлено сушествование параметров $\alpha_{\text {кр }}$ и $\gamma$ (см. ниже) таких, что при некоторых их значениях сушествуют $0,1,2$-связанные состояния системы.

Пусть $Z$ - одномерная целочисленная решетка. Обозначим через $\mathbf{S}_{m}=\left(S_{m}^{x}, S_{m}^{y}, S_{m}^{z}\right)$ оператор атомного спина величины $s=1 / 2$ узла $m$. Положим $S_{m}^{ \pm}=S_{m}^{x} \pm i S_{m}^{y}$. Обозначим через $\varphi_{0}$ вектор, называемый вакуумным, однозначно определяемый условиями $S_{m}^{+} \varphi_{0}=0, S_{m}^{z} \varphi_{0}=\frac{1}{2} \varphi_{0},\left\|\varphi_{0}\right\|=1$. Векторы $S_{m}^{-} S_{n}^{-} \varphi_{0}$ описывают состояние систе- 
мы двух магнонов, находящихся в узлах $m$ и $n$, со значением спина $s=1 / 2$. Векторы $\left\{S_{m}^{-} S_{n}^{-} \varphi_{0}\right\}$ образуют ортогональную систему. Замыкание пространства, образованного всевозможными линейными комбинациями этих векторов, обозначим через $\mathcal{H}_{2}$.

Гамильтониан рассматриваемой системы имеет вид

$$
H=-J_{z} \sum_{m, \tau}\left(S_{m}^{z} S_{m+\tau}^{z}+\alpha S_{m}^{z} S_{m+2 \tau}^{z}\right)-J_{x} \sum_{m, \tau}\left(S_{m}^{+} S_{m+\tau}^{-}+\alpha S_{m}^{+} S_{m+2 \tau}^{-}\right)
$$

и действует в пространстве $\mathcal{H}=\bigotimes_{t \in Z} \mathbb{C}_{t}^{2}$ - бесконечном тензорном произведении двумерных комплексных пространств $\mathbb{C}^{2}$, где $\mathbb{C}_{t}^{2} \equiv \mathbb{C}^{2}, \forall t \in Z$. Здесь $J_{z}>0$ и $J_{x}>0$ - параметры билинейного обменного взаимодействия ближайших соседей: $\alpha(0 \leq \alpha \leq 1)$ отношение параметра билинейного обменного взаимодействия вторых соседей к параметру билинейного обменного взаимодействия ближайших соседей: $\tau$ суммирует по \pm 1 .

ПРЕДЛОЖЕНИЕ 1. Пространство $\mathcal{H}_{2}$ инвариантно относительно оператора $H$. Oператор $\mathrm{H}_{2}=H / \mathcal{H}_{2}$ определяет ограниченный самосопряжснный оператор и унитарно-эквивалентен оператору $\bar{H}_{2}$, действующему в пространстве $l_{2}(Z \times Z)$ по формуле

$$
\begin{aligned}
\left(\bar{H}_{2} f\right)(p ; q)=- & J_{z} \sum_{\tau} \sum_{p, q} \sum_{k=1}^{2}\left\{\alpha_{k}\left(\delta_{p, q+k \tau}+\delta_{p+k \tau, q}-2\right)\right\} f(p ; q)- \\
& -J_{x} \sum_{\tau} \sum_{p, q} \sum_{k=1}^{2}\left\{\alpha _ { k } \left[-\delta_{p-k \tau, q} f(p-k \tau, q)-\right.\right. \\
& \left.\left.-\delta_{p, q-k \tau} f(p ; q-k \tau)+f(p-k \tau ; q)+f(p ; q-k \tau)\right]\right\},
\end{aligned}
$$

здесь $\alpha_{1}=1, \alpha_{2}=\alpha$.

Спектр оператора $H$ в пространстве $\mathcal{H}$ устроен очень сложно. Поэтому мы изучим его спектр в инвариантном подпространстве $\mathcal{H}_{2} \subset \mathcal{H}$. Из предложения 1 следует, что для этого достаточно изучить спектр оператора $\bar{H}_{2}$ в $l_{2}(Z \times Z)$.

Спектр оператора $\bar{H}_{2}$ легче изучается в его импульсном представлении. С этой целью обозначим через $\mathcal{F}$ преобразование Фурье:

$$
\mathcal{F}: \quad l_{2}(Z \times Z) \rightarrow L_{2}(T \times T) .
$$

Здесь $T=[0 ; 2 \pi)$ - одномерный тор, снабженный нормированной мерой Лебега $d \lambda$ : $\lambda(T)=1$. Положим $\widetilde{H}_{2}=\mathcal{F} \bar{H}_{2} \mathcal{F}^{-1}$.

ПРЕДЛОЖЕНИЕ 2. Оператор $\widetilde{H}_{2}$ в пространстве $L_{2}(T \times T)$ определяет ограниченный самосопряжснный оператор и действует по формуле

$$
\left(\widetilde{H}_{2} f\right)(x ; y)=h(x ; y) f(x ; y)-\int_{T} h_{1}(x ; y ; t) f(t ; x+y-t) d t,
$$

әде $h_{1}(x ; y ; t)=h(x ; y)+h_{2}(x ; y ; t)$, здесь

$$
\begin{aligned}
h(x ; y)= & 4 J_{z}(1+\alpha)-4 J_{x}\left[\cos \frac{x+y}{2} \cos \frac{x-y}{2}+\alpha \cos (x+y) \cos (x-y)\right], \\
h_{2}(x ; y ; t)=- & 4 J_{x}\left[\cos \frac{x+y}{2} \cos \frac{x+y-2 t}{2}+\alpha \cos (x+y) \cos (x+y-2 t)\right]+ \\
& +4 J_{z}\left[\cos \frac{x-y}{2} \cos \frac{x+y-2 t}{2}+\alpha \cos (x-y) \cos (x+y-2 t)\right] .
\end{aligned}
$$


Одной из важнейших задач математической физики является нахождение связанных состояний оператора $\mathrm{H}_{2}$ и значения энергий этих связанных состояний.

Пусть $\widetilde{\mathcal{H}}_{2}=\mathcal{F H}_{2}$. Рассмотрим многообразие $\Gamma_{\Lambda}=\{x, y \in T: x+y=\Lambda\}$. Следующее предложение важно для дальнейших исследований спектра оператора $\widetilde{H}_{2}$.

ПРЕДЛОЖЕНИЕ 3. Пусть фиксирован полньй квазиимпульс $x+y=\Lambda$. Тогда

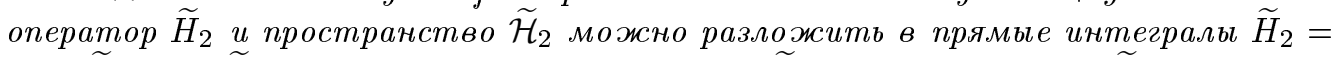
$\oplus \int_{T} \widetilde{H}_{2 \Lambda} d \Lambda, \widetilde{\mathcal{H}}_{2}=\oplus \int_{T} L_{2}\left(\Gamma_{\Lambda}\right) d \Lambda$ операторов $\widetilde{H}_{2 \Lambda}$ и пространств $\widetilde{\mathcal{H}}_{2 \Lambda}=L_{2}\left(\Gamma_{\Lambda}\right)$ так, что пространство $\widetilde{\mathcal{H}}_{2 \Lambda}$ инвариантно относительно оператора $\widetilde{H}_{2 \Lambda}$, а оператор $\widetilde{H}_{2 \Lambda}$ в $\widetilde{\mathcal{H}}_{2 \Lambda}$ действует по формуле

$$
\left(\widetilde{H}_{2 \Lambda} f_{\Lambda}\right)(x)=h_{\Lambda}(x) f_{\Lambda}(x)-\int_{T} h_{1 \Lambda}(x ; t) f_{\Lambda}(t) d t
$$

здесь $h_{\Lambda}(x)=h(x ; \Lambda-x), \quad h_{1 \Lambda}(x ; t)=h_{1}(x ; \Lambda-x ; t) u f_{\Lambda}(x)=f(x ; \Lambda-x)$.

Рассмотрим оператор $K_{\Lambda}$ :

$$
\left(K_{\Lambda}(z) f_{\Lambda}\right)(x)=-\int_{T} \frac{h_{1 \Lambda}(x ; t)}{h_{\Lambda}(t)-z} f_{\Lambda}(t) d t .
$$

Он является вполне непрерывным в пространстве $\widetilde{\mathcal{H}}_{2 \Lambda}$ для значений $z$, лежаших вне множества $G_{\Lambda}=\operatorname{Im} h_{\Lambda}(x)$. Обозначим через $\Delta_{\Lambda}(z)$ определитель Фредгольма оператора $E(\Lambda)+K_{\Lambda}(z)\left(E_{\Lambda}-\right.$ единичный оператор в $\left.\mathcal{H}(\Lambda)\right)$ :

$$
\Delta_{\Lambda}(z)=1+\sum_{n=1}^{\infty} \frac{1}{n !} d_{n \Lambda}(z)
$$

где

$$
\begin{aligned}
& d_{n \Lambda}(z)=\int_{T} \cdots \int_{T} \frac{K_{\Lambda z}\left(\begin{array}{c}
t_{1}, \ldots, t_{n} \\
t_{1}, \ldots, t_{n}
\end{array}\right)}{\prod_{j=1}^{n}\left(h_{\Lambda}\left(t_{j}\right)-z\right)} d t_{1} \ldots d t_{n},
\end{aligned}
$$

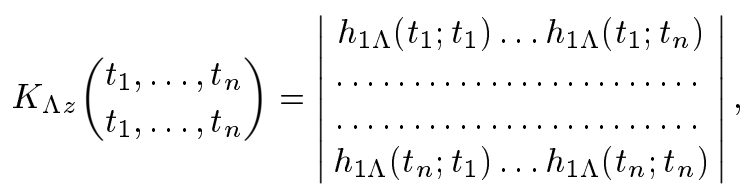

$$
\begin{aligned}
& h_{1 \Lambda}\left(t_{k} ; t_{j}\right)=4 J_{z}(1+\alpha)-4 J_{x}\left[\cos \frac{\Lambda}{2} \cos \left(\frac{\Lambda}{2}-t_{k}\right)+\alpha \cos \Lambda \cos \left(\Lambda-2 t_{k}\right)\right]- \\
& -4 J_{x}\left[\cos \frac{\Lambda}{2} \cos \left(\frac{\Lambda}{2}-t_{j}\right)+\alpha \cos \Lambda \cos \left(\Lambda-2 t_{j}\right)\right]+ \\
& +4 J_{z}\left[\cos \left(\frac{\Lambda}{2}-t_{j}\right) \cos \left(\frac{\Lambda}{2}-t_{k}\right)+\alpha \cos \left(\Lambda-2 t_{j}\right) \cos \left(\Lambda-2 t_{k}\right)\right] \text {. }
\end{aligned}
$$

Общий вид функции $\Delta_{\Lambda}(z)$ довольно громоздок, поэтому мы его здесь не приводим.

Заметим, что непрерывный спектр оператора $\widetilde{H}_{2 \Lambda}$ состоит из множества значений функции $h_{\Lambda}(x)$. 
Лемма. Число $z \bar{\epsilon} G_{\Lambda}$ - собственное значение оператора $\widetilde{H}_{2 \Lambda}$ тогда и только тогда, когда оно является решением уравнения $\Delta_{\Lambda}(z)=0$.

ДокАЗАТЕЛЬСтво. Пусть $z=z_{0}$ - собственное значение оператора $\widetilde{H}_{2 \Lambda}$. Это означает, что число $\mu=-1$ является собственным значением оператора $K_{\Lambda}\left(z_{0}\right)$, т.е. однородное уравнение

$$
f_{\Lambda}(x)-\int_{T} \frac{h_{1 \Lambda}(x ; t)}{h_{\Lambda}(t)-z} f_{\Lambda}(t) d t=0
$$

имеет нетривиальное решение. Следовательно, в точке $z=z_{0}$ функция $\Delta_{\Lambda}(z)$ равна нулю: $\Delta_{\Lambda}\left(z_{0}\right)=0$.

Пусть теперь точка $z=\bar{z}$ является нулем функции $\Delta_{\Lambda}(z): \Delta_{\Lambda}(\bar{z})=0$. Тогда однородное уравнение

$$
f_{\Lambda}(x)-\int_{T} \frac{h_{1 \Lambda}(x ; t)}{h_{\Lambda}(t)-z} f_{\Lambda}(t) d t=0
$$

имеет нетривиальное решение. Это означает, что число $z=z_{0}$ есть собственное значение оператора $K_{\Lambda}(\bar{z})$. Отсюда следует, что число $z=\bar{z}$ - собственное значение оператора $\widetilde{H}_{2 \Lambda}$.

ОПРЕДЕЛЕниЕ. Собственная функция $\varphi_{\Lambda}$ оператора $\widetilde{H}_{2 \Lambda}$, отвечаюшая собственному значению $z_{\Lambda}$, называется связанным состоянием оператора $\widetilde{H}_{2}$, а величина $z_{\Lambda}$ - энергией этого связанного состояния.

Пусть теперь $\Lambda=\pi$. Тогда имеет место следующее предложение.

ПРЕДЛОЖЕНИЕ 4.

А. При условии $0<\alpha<\frac{J_{z}}{2 J_{x}}$ уравнение $\Delta_{\pi}(z)=0$ имеет ровно два решения:

$$
\begin{aligned}
& z_{\pi}^{\prime}=4 J_{z}+2 \alpha J_{z}-\frac{2 \alpha J_{x}^{2}}{J_{z}} \\
& z_{\pi}^{\prime \prime}=4 J_{z}+4 \alpha\left(J_{z}+J_{x}\right)-\frac{2 J_{z}^{2}}{J_{z}-\alpha J_{x}}
\end{aligned}
$$

которые лежат ниже значений $m_{\pi}$, әде $m_{\pi}=\inf _{x} h_{\pi}(x)$. (Заметим, что $z_{\pi}^{\prime} u$ $z_{\pi}^{\prime \prime}$ совпадают при $\alpha=J_{z} /\left(J_{x}+J_{z}\right)$ u $\alpha=J_{z}^{2} /\left[J_{x}\left(J_{x}+J_{z}\right)\right]$, а при остальных значениях $\alpha$ они различны.)

Б. При условии $\alpha \geq J_{z} /\left(2 J_{x}\right)$ уравнение $\Delta_{\pi}(z)=0$ имеет единственное решение $z=z_{\pi}^{\prime}<m_{\pi}$.

Из предложения 4 и леммы вытекает следующая теорема.

TEOPEMA 1

А. Если выполняется условие "А" предложения 4, то оператор $\widetilde{H}_{2}$ при $\Lambda=\pi$ имеет ровно два связанных состояния $\varphi_{\pi}^{\prime}$ и $\varphi_{\pi}^{\prime \prime}$ со значениями әнергии $z=z_{\pi}^{\prime} u$ $z=z_{\pi}^{\prime \prime}$, соответственно. Эти значения әнергии лежат ниже значений $m_{\pi}$.

Б. Если выполняется условие "Б" предложения 4, то оператор $\widetilde{H}_{2}$ при $\Lambda=\pi$ имеет единственное связанное состояние $\varphi_{\pi}^{\prime}$ со значением энергии $z=z_{\pi}^{\prime}<m_{\pi}$. В этом случае второе связанное состояние исчезает, а его әнергия поглощается непрерывным спектром оператора $\widetilde{H}_{2 \pi}$. 
ДокАЗАТЕЛЬСтво. Подробный анализ функции $\Delta_{\Lambda}(z)$ показьвает, что уравнение $\Delta_{\pi}(z)=0$ эквивалентно уравнению

$$
\begin{gathered}
\left(1-4 J_{z} \int_{T} \frac{\sin ^{2} s d s}{h_{\pi}(s)-z}\right)\left\{\int_{T} \frac{d s}{h_{\pi}(s)-z}\left(1-\int_{T} \frac{\cos 2 s\left(4 \alpha J_{z} \cos 2 s-4 \alpha J_{x}\right) d s}{h_{\pi}(s)-z}\right)+\right. \\
\left.+\int_{T} \frac{\cos 2 s d s}{h_{\pi}(s)-z} \int_{T} \frac{4 \alpha J_{z} \cos 2 s-4 \alpha J_{x}}{h_{\pi}(s)-z} d s\right\}=0
\end{gathered}
$$

где

$$
h_{\pi}(s)=4 J_{z}-4 \alpha J_{x} \cos 2 s .
$$

Интегралы, содержащиеся в этом уравнении, вычисляются в квадратурах. Приравнивая к нулю первый сомножитель и вычисляя интегралы для значений $z$, лежащих ниже значений непрерывного спектра оператора $\widetilde{H}_{2 \Lambda}$, получим следующее уравнение:

$$
1-\frac{J_{z}}{2 \alpha J_{x}}+\frac{J_{z}\left(4 \alpha J_{z}+4 J_{z}-4 \alpha J_{x}\right)-z}{2 \alpha J_{x} \sqrt{\left(4 J_{z}+4 \alpha J_{z}-z\right)^{2}-16 \alpha^{2} J_{x}^{2}}}=0 .
$$

Исследование показывает, что это уравнение имеет ровно одно решение $z_{\pi}^{\prime \prime}$ при $0<\alpha<$ $J_{z} /\left(2 J_{x}\right)$ :

$$
z_{\pi}^{\prime \prime}=4 J_{z}+4 \alpha J_{z}+4 \alpha J_{x}-2 J_{z}^{2} /\left(J_{z}-\alpha J_{x}\right) .
$$

Далее, приравнивая к нулю второй сомножитель уравнения (1) и вычисляя интегралы, получим уравнение

$$
\frac{J_{z}}{4 \alpha J_{x}^{2}}-\frac{J_{z}\left(4 J_{z}+4 \alpha J_{z}-z\right)-4 \alpha J_{x}^{2}}{4 \alpha J_{x}^{2} \sqrt{\left(4 J_{z}+4 \alpha J_{z}-z\right)^{2}-16 \alpha^{2} J_{x}^{2}}}=0 .
$$

Исследование этого уравнения показывает, что оно имеет ровно одно решение $z_{\pi}^{\prime}$ при всех значениях параметра $\alpha(0 \leq \alpha \leq 1)$ :

$$
z_{\pi}^{\prime}=4 J_{z}+2 \alpha J_{z}-\left(2 \alpha J_{x}^{2}\right) / J_{z}
$$

Предложение 4 доказано.

Заметим, что когда значение параметра $\alpha$ возрастая переходит через значения $J_{z} /\left(2 J_{x}\right)$, одно из связанных состояний оператора $\widetilde{H}_{2}$ исчезает, а его энергия поглощается непрерывным спектром оператора $\widetilde{H}_{2 \Lambda}$.

Пусть теперь $\Lambda=\pi / 2$. В этом случае имеет место следующее предложение.

ПРЕДЛОЖЕНИЕ 5 .

А. При условии $J_{x}<\sqrt{2} J_{z}$ и $J_{x} \neq J_{z}$ уравнение $\Delta_{\pi / 2}(z)=0$ имеет ровно два решения $z_{\pi / 2}^{\prime} \quad$ u $z_{\pi / 2}^{\prime \prime}$ для всех значений параметра $\alpha \quad(0 \leq \alpha \leq 1) ; \quad z_{\pi / 2}^{\prime}, z_{\pi / 2}^{\prime \prime}<$ $m_{\pi / 2}=\inf _{x} h_{\pi}(x)$.

Б. При $J_{z}=J_{x}$ (изотропный случай) уравнение $\Delta_{\pi / 2}(z)=0$ имеет единственное решение $z=z_{\pi / 2}^{\prime}$ для всех значений параметра $\alpha \quad(0 \leq \alpha \leq 1)$. Это решение лежит ниже значений $m_{\pi / 2}$.

В. Если $J_{x} \geq \sqrt{2} J_{z}$, то существует такое значение параметра $\alpha$, обозначаемое $\alpha_{\mathrm{Kp}}$, что при $0<\alpha<\alpha_{\mathrm{Kр}}$ уравнение $\Delta_{\pi / 2}(z)=0$ имеет ровно два решения $z=z_{\pi / 2}^{1} u z=z_{\pi / 2}^{2}$, а при $\alpha=\alpha_{\text {кр }}$ имеет ровно одно решение $z=z_{\pi / 2}^{1}$. Если же $\alpha>\alpha_{\mathrm{kp}}$, уравнение $\Delta_{\pi / 2}(z)=0$ не имеет решений ниже значений $m_{\pi / 2}$. Заметим, что $\alpha_{\mathrm{кр}}$ зависит от $J_{x}, J_{z}$ и $\alpha_{\mathrm{\kappa р}} \in[0 ; 1]$. Например, при $J_{x}=\sqrt{2} J_{z}$ имеем $\alpha_{\mathrm{\kappa p}} \approx 0,38$. 
ДокАЗАТЕЛЬство. Нетрудно показать, что уравнение $\Delta_{\pi / 2}(z)=0$ эквивалентно уравнению

$$
\begin{aligned}
\alpha J_{z}\left[\left(4 J_{z}\right.\right. & \left.\left.+4 \alpha J_{z}-z\right) \sqrt{\left(4 J_{z}+4 \alpha J_{z}-z\right)^{2}-8 J_{x}^{2}}-\left(4 J_{z}+4 \alpha J_{z}-z\right)^{2}+4 J_{x}^{2}\right]= \\
= & 2 J_{x}^{2}\left[J_{z}+2\left(J_{z}^{2}-J_{x}^{2}\right) /\left(2 J_{z}+4 \alpha J_{z}-z\right)-\right. \\
& \left.-\left\{J_{z} /\left(2 J_{z}+4 \alpha J_{z}-z\right)\right\} \sqrt{\left(4 J_{z}+4 \alpha J_{z}-z\right)^{2}-8 J_{x}^{2}}\right] .
\end{aligned}
$$

Исследуя это уравнение при $z<m_{\pi / 2}$, получим доказательство предложения 5.

Из предложения 5 и леммы следует теорема.

TEOPEMA 2.

А. Если выполняется условие "А" предложения 5 и $\Lambda=\pi / 2$, то при всех значениях параметра $\alpha$ оператор $\widetilde{H}_{2}$ имеет ровно два связанньх состояния $\varphi_{\pi / 2}^{\prime}$ и $\varphi_{\pi / 2}^{\prime \prime}$ со значениями әнергии $z=z_{\pi / 2}^{\prime}, z_{\pi / 2}^{\prime \prime}<m_{\pi / 2}$.

Б. Если выполняется условие "Б" предложсения 5 и $\Lambda=\pi / 2$, то при всех значениях параметра а оператор $\widetilde{H}_{2}$ имеет единственное связанное состояние $\varphi_{\pi / 2}^{\prime}$ со значением әнергии $z=z_{\pi / 2}^{\prime}<m_{\pi / 2}$.

В. Если выполняется условие "В" предложения 5 и $\Lambda=\pi / 2$, то при $\alpha \in\left[0 ; \alpha_{\mathrm{\kappa p}}\right)$ оператор $\widetilde{H}_{2}$ имеет ровно два связанньх состояния $\varphi_{1}$ и $\varphi_{2}$ со значениями энергий $z=z_{\pi / 2}^{1}, z_{\pi / 2}^{2}<m_{\pi / 2}$. При $\alpha=\alpha_{\mathrm{kp}}$ оператор $\widetilde{H}_{2}$ имеет единственное связанное состояние со значением әнергии $z=z_{\pi / 2}^{1}<m_{\pi / 2}$. При $\alpha>\alpha_{\mathrm{\kappa p}}$ оператор $\widetilde{H}_{2}$ не имеет ни одного связанного состояния.

Пусть теперь $\Lambda=0$. В этом случае имеет место следуюшее предложение.

ПРЕДЛОЖЕНИЕ 6. При всех значениях $\alpha, J_{z}$ и $J_{x}$ уравнение $\Delta_{\Lambda=0}(z)=0$ не имеет ни одного решения ниже непрерывного спектра оператора $\widetilde{H}_{2 \Lambda}$.

ДОКАЗАТЕЛЬСТВО предложения 6 аналогично доказательству предложений 4 и 5.

Из предложения 6 вытекает теорема.

ТеОрема 3. Пусть $\Lambda=0$. Тогда оператор $\widetilde{H}_{2}$ при всех значениях параметров $\alpha, J_{x}$ и $J_{z}$ не имеет связанных состояний.

В области значений полного квазиимпульса $\Lambda,-\pi<\Lambda<\pi, \Lambda \neq \frac{\pi}{2}$ и $\Lambda \neq 0$, исследования связанного состояния оператора $\widetilde{H}_{2}$ показывают, что при фиксированном значении полного квазиимпульса $\Lambda$ существует положительное вешественное число $\gamma$, зависяшее только от $\Lambda$, такое, что при $J_{x}<\gamma J_{z}$ и при всех значениях параметра $\alpha$ оператор $\widetilde{H}_{2}$ имеет ровно два связанных состояния. При условии $J_{x} \geq \gamma J_{z}$ имеются два связанных состояния в случае, если $0<\alpha<\alpha_{\text {кр }}$, одно связанное состояние, если $\alpha=\alpha_{\text {кр }}$ и не сушествует ни одного связанного состояния, если $\alpha>\alpha_{\text {кр }}$. Энергия этих связанных состояний всегда меньше $m_{\Lambda}=\inf _{x} h_{\Lambda}(x)$. При условии $J_{x}=J_{z}$ (изотропный случай) сушествует одно связанное состояние, если $0<\alpha<\alpha_{\text {кр }}$, и не сушествует ни одного связанного состояния, если $\alpha \geq \alpha_{\mathrm{kp}}$.

Отметим, что если связанное состояние системы исчезает при некоторых значениях параметров $J_{x}, J_{z}$ и $\alpha$, то оно появится при некоторых других значениях. 
Таким образом, при $-\pi<\Lambda<\pi, \Lambda \neq \frac{\pi}{2}, \Lambda \neq 0$ могут существовать два или одно связанное состояние системы или же не существует ни одного связанного состояния со значениями энергии, меньшими $m_{\Lambda}$. Отметим, что в этом случае $\alpha_{\text {кр }}=\alpha\left(\Lambda, J_{z}, J_{x}\right)$. Коротко приводим доказательство этого утверждения.

Заметим, что все интегралы, содержашиеся в уравнении $\Delta_{\Lambda}(z)=0$ для значений $z<m_{\Lambda}$, явно вычисляются в квадратурах и их значения зависят от параметров $J_{x}, J_{z}$, $\alpha$ и $\Lambda$. Выражение для $\Delta_{\Lambda}(z)$ получается громоздким.

Уравнение $\Delta_{\Lambda}(z)=0$ после несложных преобразований можно свести к некоторому алгебраическому уравнению

$$
F\left(J_{x}, J_{z}, \alpha, \Lambda, z\right)=0
$$

Исследование этого алгебраического уравнения показывает, что если $J_{x}<\gamma J_{z}$, то при всех значениях $\Lambda$ уравнение (2) имеет ровно два решения. Если же $J_{x} \geq \gamma J_{z}$, то уравнение (2) имеет ровно два решения только в том случае, если $0<\alpha<\alpha_{\text {кр }}$. Если $\alpha=\alpha_{\text {кр }}$, уравнение (2) имеет единственное решение. Причем все решения $z$ уравнения (2) имеют значения меньше $m_{\Lambda}$. Если же $\alpha>\alpha_{\text {кр }}$, то не сушествует решения уравнения (2) со значениями меньше $m_{\Lambda}$.

Следовательно, при указанных выше соотношениях между параметрами $J_{x}, J_{z}, \alpha$ и $\gamma$ оператор $\widetilde{H}_{2 \Lambda}$ имеет $0,1,2$-связанные состояния со значениями энергии, меньшими $m_{\Lambda}$.

\section{Список литературы}

[1] Bethe H. A. // Z. Phys. 1931. B. 71. S. 205.

[2] Fukuda N., Wortis M. // J. Phys. Chem. Solids. 1963. V. 24. P. 1675-1677.

[3] Wortis M. // Phys. Rew. 1963. V. 132. № 1. P. 85-97.

[4] Majumdar C. K. // J. Math. Phys. 1969. V. 10. P. 117.

[5] Ono J., Mikado S., Oguchi T. // J. Phys. Soc. Japan. 1971. V. 30. № 8. P. 358

[6] Гочев И. Г. // ТМФ. 1973. Т. 15. №1. С. 120-126.

[7] Гочев И. Г. // ЖЭТФ. 1971. Т. 61. Вып. 4(10). С. 1674.

[8] Изюмов Ю. А., Скрябин Ю. Н. Статистическая механика магнитоупорядоченных систем. М.: Наука., 1987.

"Кибернетика" АН РУз

Поступила в редакцию

8.VIII.1994 г., после доработки

16.XI.1995 г

\section{S. M. Tashpulatov \\ EXPLORATION OF SPECTRUM OF THE ENERGY \\ OPERATOR OF TWO-MAGNON SYSTEM IN THE ONE-DIMENSIONAL ANISOTROPIC HEISENBERG FERROMAGNET WITH INTERACTION OF SECOND NEIGHBOURS}

We investigate a physical system of two magnons in the one-dimensional anisotropic Heisenberg ferromagnet with the interaction up to two neighbours. We study the spectrum and bound states of the Hamiltonian of the two-magnon system. It is established that in some cases additional bound states can appear or disappear in comparison with the isotropic case. 